\title{
PERI-IMPLANT BONE LOSS AND ESTHETIC OUTCOME OF SINGLE-TOOTH IMPLANT IN PATIENTS WITH TYPE 2 DIABETES
}

\author{
Walid Al-Zordk*, Sally Awad** and Samah Saker ${ }^{* * *}$
}

\begin{abstract}
Background: Esthetic outcome associated with dental implant in type 2 diabetic patients remained uninvestigated.

Purpose: To study the impact of type 2 diabetes mellitus on clinical and esthetic outcomes of single-tooth implant following delayed implant installation in the anterior maxilla.

Materials and Methods: Forty patients were recruited for this investigation. Half of patients were previously diagnosed with controlled type 2 diabetes mellitus (group D) and the remaining $(\mathrm{n}=20)$ were healthy individuals (group H). All implants were inserted in the anterior part of the maxilla either with sufficient bone volume /or with labial bone defect. Evaluations of soft and hardtissue changes were checked during the follow-up period up to 24 months.

Results: Significant loss in marginal osseous tissue was observed during 24 months of evaluation with respect to diabetic $(\mathrm{p}=.001)$ regardless the bone augmentation procedure used, while group $\mathrm{H}$ showed significant loss at 6 months of evaluation followed by non-significant changes of the marginal bone level till 24 months. Mean PES and WES scores did not diverge significantly among groups $\mathrm{D}$ and $\mathrm{H}(\mathrm{p}>.05)$.

Conclusions: Single tooth dental implants can remain functionally and esthetically acceptable in type 2 diabetic patients in a manner similar to healthy patients provided carful controlling and maintaining of blood glycemic level.
\end{abstract}

KEY WORDS: Diabetic patient, Dental implant, Anterior maxilla, Bone augmentation.

\section{INTRODUCTION}

Dental implants are a commonly used treatment modality for management of fully and partially edentulous patients in modern dental clinics. ${ }^{1-4}$
To assure the long-term clinical success, good initial stability, healing of soft tissue immediately following implant placement, and osseointegration are crucial. ${ }^{5-8}$

\footnotetext{
* Assistant Professor, Fixed prosthodontics Department, Faculty of Dentistry, Mansoura University, Mansoura, Egypt.

** Associate Professor, Department of Oral and Maxillofacial Surgery, Faculty of Dentistry, Mansoura University, Mansoura, Egypt.

***Associate Professor, Fixed prosthodontics Department, Faculty of Dentistry, Mansoura University, Mansoura, Egypt.
} 
In the anterior maxilla, initiation and preservation of appropriate hard and soft tissue architecture are substantial to attain pleasing esthetic outcomes. Appropriate bone dimension and architecture of the alveolar edentulous ridge, comprising an intact facial bone wall of sufficient thickness and height in combination with a correct restoration-driven implant placement, are mandatory to support periimplant soft tissue. ${ }^{9-13}$

Careful patient selection with proper wound healing and without any metabolic disorders should be considered for long term clinical success of dental implant. ${ }^{14,15}$ Several medical conditions able to interfere with normal body reaction following surgical intervention which require careful evaluation after surgery. These conditions call for precise estimation but not spontaneously hinder implant therapy in every influenced individual. ${ }^{15,16}$

Diabetes mellitus is a long-term metabolic disturbance that is essentially distinguished by insulin impedance, relative insulin insufficiency, and elevated levels of blood glucose (hyperglycemia). 18, 19 Diabetic patients have increased frequency of delayed wound healing, impaired response to infection, and periodontal inflammatory conditions with subsequent tooth loss. ${ }^{15,20-22,23}$

Many researchers reported direct relation between diabetic patients and failure of dental implant. ${ }^{24-27}$ However Alasqah et al ${ }^{28}$ evaluated delayed loaded dental implant treatment in diabetic patients after a 6-year of clinical observation, they concluded that when glycemic levels of type 2 diabetic patients are carefully controlled and maintained, the prognosis of implant therapy in esthetic area can remain functionally stable and esthetically pleasant in a manner similar to healthy individuals.

Safety replacement of missing teeth with an implant, as a treatment modality, with or without osseous augmentation steps in patients' with diabetes is yet controversial. Normal wound healing following implant surgery is required for osseointegration. The host bed requires an adequate blood supply to deliver the necessary elements for wound repair and host defense. ${ }^{29,30}$ Diabetic has been shown to impair wound healing; therefore, it may also impair osseointegration. Furthermore, diabetic has been implicated in reduced bone density, possibly giving rise to poor quality bone. Poor bone quality has been attributed to implant loss. The target of this clinical investigation was to evaluate prospectively clinical and esthetic outcome of dental implant placement in esthetic zone in relation to controlled diabetic.

\section{MATERIALS AND METHODS}

\section{Study design}

This prospective, controlled clinical trial in which patients were consecutively included based on certain inclusion and exclusion specifications. After getting ethical approval from the Research Ethics Committee at faculty of dentistry, Mansoura university, forty patients were picked from the faculty outpatient clinic. The patients have been asked and accepted to participate in the research in a consecutive schedule, provided they redeem the subsequent criteria: inclusion criteria; either systemically healthy individuals (group H) or patients with controlled diabetics (type 2, group D), who are on active medical treatment and receiving oral hypoglycemic. Their previous diabetes history was confirmed with the physician's report. Preoperative Glycated hemoglobin A1c (HbA1c) levels were between $6 \%$ and $7.5 \%$. HbA1c levels of the patients were measured 1 week before surgery, 3-5 months after implant placement and during follow up interval at the same laboratory.

All the patients are in need of an implantsupported single tooth restoration within anterior maxilla, have either sufficient bone volume and /or with labial bone defect (with at least $10 \mathrm{~mm}$ osseous height but less than $5 \mathrm{~mm}$ osseous width). 
CBCT was used for every patient to check for the bone volume and aid in surgical template fabrication. (Fig. 1) Exclusion criteria: Alcohol or drug abuse, other systemic conditions which interfere with the surgical procedure, presence of any systemic contraindication other than diabetes, the individual is not able to give her/his informed consent to participate, smokers and bruxism or other destructive oral habits.

\section{Surgical interventions}

Before surgery, the patient received antibiotics (2 x 625 mg Augmentin ${ }^{\circledR}$, Galaxo Smith Kline Co). Patients were instructed to use mouthwash (Orovex, Macro-International Co). Surgery was performed under local anesthesia (Ubistesin forte, 3M ESPE). A full-thickness flab was raised followed by osteotomy preparation and implant installation (HEXACONE, IHDEDENTAL, Switzerland) following manufacturer's recommendation. (Fig. 2) In groups D2 and $\mathrm{H} 2$, the space between the fixture and the labial bone was loaded with xenograft bone substitute (tutobone; tutogen, germany) and the resorbable membrane were placed. After six months, the implant was uncovered and the healing cap was inserted. After two weeks, the prosthetic phase was started by impression making and definitive restoration was constructed.

\section{Evaluation phase}

Clinical follow-up was carried at 6 months after implant placement with definitive restorations, 12 months and finally after 24 months (Fig. 3). At each recall appointment, both clinical and periapical radiographic examinations were evaluated.

Peri-implant clinical indices: Modified plaque index $(\mathrm{mPI})^{31}: 0=$ no plaque detected, $1=$ plaque detected by running a probe across the surface.; 2 = plaque was observed visually.; 3 = abundance of plaque. The presence of plaque was detected by running a probe along the smooth marginal surface of the implant. The probing was assessed four sites around each implant (mesial, labial, distal and lingual). The sum of the four probing surfaces were calculated was divided by four to get the average score around each implant.

\section{Radiographic evaluation}

Using standard periapical radiographs, the marginal bone loss (MBL) was evaluated at 6 months, 12 months and finally after 24 months. The changes in the marginal bone around implant were measured according to the following: ${ }^{32}$ Two reference points (one mesial and one distal) at implant / abutment junction was used as reference points. A straight line was established between the two reference points which represent the baseline height. Mesial and distal perpendicular lines were created running from the line that represented baseline height to the most coronal point of bone contact. MBL was calculated by subtracting the two measurements. Both mesial bone loss and distal bone loss were determined; the larger of the two values was considered the bone loss of the implant in question.

\section{Esthetic evaluation}

Assessment of esthetic results were rated employing pink esthetic score (PES) and white esthetic score (WES) ${ }^{33-35}$. A front and occlusal color views photographs was taken for every case with high resolution camera and the images were then transported to a computer with an enhanced resolution screen for screening and estimation. All clinical measurements were evaluated by a blinded, expert examiner.

\section{Statistical Methods}

In order to perform the descriptive statistical analysis, all data were entered in a Microsoft Excel 2010 worksheet. For further data analysis statistical package for social science version 20 SPSS (SPSS, IBM Corp., New York) was used. For mPI,PES/ WES Wilcoxon signed rank test was used while two way mixed analysis of variance (ANOVA) was used for analysis of MBL at 5\% level of significant. 


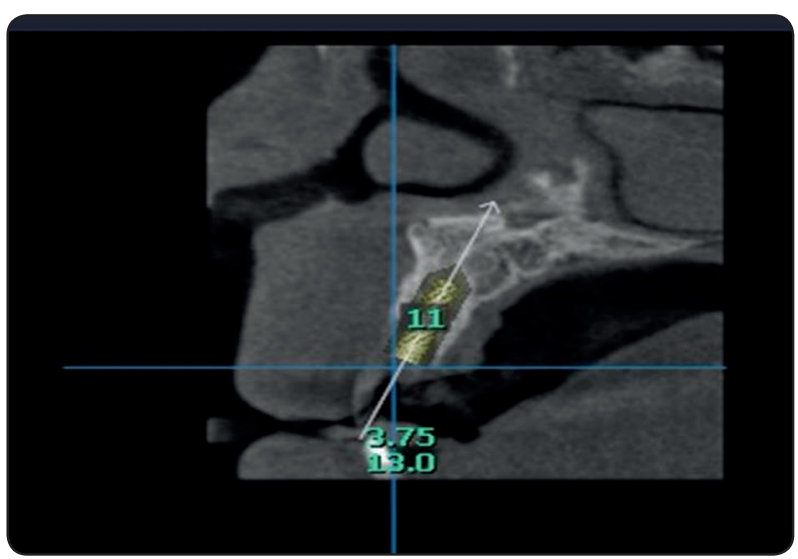

Fig. (1) CBCT scan used to plan the implant location.

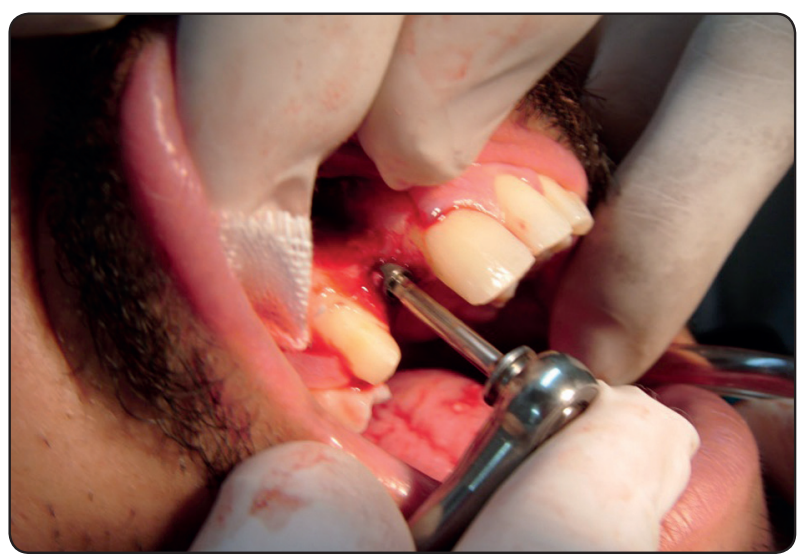

Fig. (2) Photograph representing implant installation showing labial bone defect.

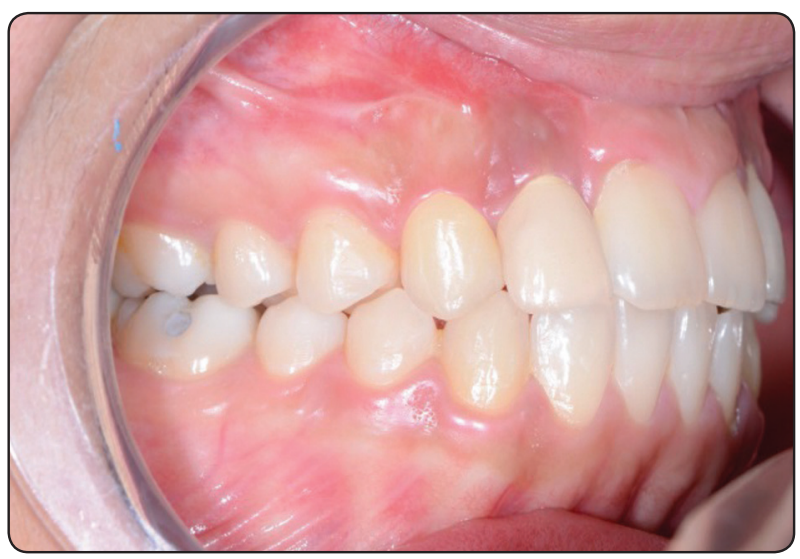

Fig. (3) Photograph at 24 months (group D1).

\section{RESULTS}

With 40 implants, our study had 40 patients (18 men, 22 women) with a mean age of $40 \pm 7$ years at the time of implant placement (table. 1). The mean time from surgery to evaluation was 30 months starting from August 2016 till November 2018. At the end of evaluation period, no implants were lost through the observation interval and all fixtures were well integrated giving rise to a $100 \%$ implant survival after 24 months of function after implant placement. The biological integration of all implants was excellent with no biological complications at implant sites.

TABLE (1) Mean age and gender of each group under study.

\begin{tabular}{|l|l|r|r|r|}
\hline Groups & Gender & \multicolumn{1}{|c|}{ Mean } & \multicolumn{1}{|c|}{$\begin{array}{c}\text { Std. } \\
\text { Deviation }\end{array}$} & N \\
\hline $\begin{array}{l}\text { Healthy without } \\
\text { augmentation } \\
\text { (H1) }\end{array}$ & male & 37.1 & 4.8 & 6 \\
\cline { 2 - 5 } & female & 40 & 6.2 & 4 \\
\hline $\begin{array}{l}\text { Healthy with } \\
\text { augmentation } \\
\text { (H2) }\end{array}$ & male & 38.7 & 4.5 & 3 \\
\cline { 2 - 5 } & female & 42 & 8.4 & 7 \\
\hline $\begin{array}{l}\text { Diabetic without } \\
\text { augmentation } \\
\text { (D1) }\end{array}$ & male & 39.6 & 5.2 & 5 \\
\cline { 2 - 5 } & female & 40.4 & 7.3 & 5 \\
\hline $\begin{array}{l}\text { Healthy with } \\
\text { augmentation } \\
\text { (D2) }\end{array}$ & male & 44 & 9.9 & 4 \\
\cline { 2 - 5 } & female & 41.5 & 6.6 & 6 \\
\hline
\end{tabular}

\section{Plaque index}

All groups have minimal rise in the proportion of plaque during 24 months of evaluation and the difference between study groups was insignificant (table 2). Also, there were no significant differences for probing depth among all study groups.

\section{Marginal level bone changes}

The marginal bone loss was measured and the means of MBL was obtained at 6 months, one 
year and two years. The mean values of MBL are represented in table 3 at each time interval. When comparing implants placed in controlled diabetic versus implants placed in healthy individuals, it was found that, the level of marginal bone surrounding implants in controlled diabetic with and without bone augmentation decreased by $0.74 \pm 0.11$ and $1 \pm 0.16 \mathrm{~mm}$ respectively, while in healthy individuals the decreased was $0.62 \pm 0.07$ and $0.45 \pm 0.08 \mathrm{~mm}$ in groups $\mathrm{H} 1$ and $\mathrm{H} 2$ respectively and the difference was statistically significant ( $\mathrm{p}=0.001)$.

Overall evaluation period, the bone loss in controlled diabetic patients were significantly greater $(\mathrm{p}=0.001)$ than that demonstrated surrounding the implants in healthy individuals regardless the augmentation procedure.

\section{PES/WES}

The detailed PES/WES scores of the 40 examined single-tooth implants are showed in Table 4 . The mean total PES was for healthy individuals with and without bone augmentation was 7.3 and 7.2 respectively, while for controlled diabetic patient was 6.4 and 6.3 for respectively. For the WES, the mean total was $8.7,8.2$ for healthy individuals and 8.6 and 8 controlled diabetic patient at 6 months (table 4). Figures $2 \& 3$ are the clinical photographs and the radiographs of representative example of central incisor single-tooth implant with a total PES/ WES of 17 (corresponding to an excellent overall esthetic outcome)

TABLE (2) Mean and SD of plaque index of each group under study.

\begin{tabular}{|c|c|c|c|c|c|c|}
\hline $\begin{array}{l}\text { Evaluation } \\
\text { period }\end{array}$ & & H1 & $\mathrm{H} 2$ & D1 & D2 & \\
\hline \multirow{5}{*}{ after 6} & Mean & 0.10 & 0.10 & 0.10 & 0.10 & \multirow{5}{*}{1.00} \\
\hline & Median & 0 & 0 & 0 & 0 & \\
\hline & S D & 0.31 & 0.31 & 0.31 & 0.31 & \\
\hline & Minimum & 0 & 0 & 0 & 0 & \\
\hline & Maximum & 1.00 & 1.00 & 1.00 & 1.00 & \\
\hline \multirow{5}{*}{ one year } & Mean & 0.30 & 0.30 & 0.20 & 0.20 & \multirow{5}{*}{.76} \\
\hline & Median & 0 & 0 & 0 & 0 & \\
\hline & S D & 0.48 & 0.48 & 0.42 & 0.42 & \\
\hline & Minimum & 0 & 0 & 0 & 0 & \\
\hline & Maximum & 1 & 1 & 1 & 1 & \\
\hline \multirow{5}{*}{ two years } & Mean & 0.40 & 0.60 & 0.80 & 0.50 & \multirow{5}{*}{.51} \\
\hline & Median & 0 & 0 & 0 & 0 & \\
\hline & S D & 0.69 & 0.96 & 1.13 & 0.71 & \\
\hline & Minimum & 0 & 0 & 0 & 0 & \\
\hline & Maximum & 2 & 3 & 3 & 2 & \\
\hline
\end{tabular}


TABLE (3) Mean and SD of marginal bone loss of each group under study.

\begin{tabular}{|c|c|c|c|c|c|c|}
\hline & Groups & $\mathrm{N}$ & Mean & Std. Deviation & $\mathrm{p}$ & $\mathrm{p}$ \\
\hline \multirow[t]{4}{*}{ after 6} & $\mathrm{H} 1$ & 10 & 0.62 & 0.07 & \multirow[t]{2}{*}{.001} & \multirow{4}{*}{.001} \\
\hline & $\mathrm{H} 2$ & 10 & 0.45 & 0.08 & & \\
\hline & D1 & 10 & 1.00 & 0.16 & \multirow[t]{2}{*}{.001} & \\
\hline & D2 & 10 & 0.74 & 0.11 & & \\
\hline \multirow[t]{4}{*}{ one year } & H1 & 10 & 0.96 & 0.11 & \multirow[t]{2}{*}{.016} & \multirow{4}{*}{.001} \\
\hline & $\mathrm{H} 2$ & 10 & 0.83 & 0.08 & & \\
\hline & D1 & 10 & 1.35 & 0.09 & \multirow[t]{2}{*}{.00} & \\
\hline & D2 & 10 & 1.11 & 0.12 & & \\
\hline \multirow[t]{4}{*}{ two years } & $\mathrm{H} 1$ & 10 & 1.19 & 0.10 & \multirow[t]{2}{*}{.42} & \multirow{4}{*}{.001} \\
\hline & $\mathrm{H} 2$ & 10 & 1.16 & 0.09 & & \\
\hline & D1 & 10 & 1.61 & 0.09 & \multirow[t]{2}{*}{.001} & \\
\hline & D2 & 10 & 1.32 & 0.03 & & \\
\hline
\end{tabular}

TABLE 4. Mean PES/WES of the study groups.

\begin{tabular}{|c|c|c|c|c|c|c|c|c|c|c|c|c|c|c|c|}
\hline & & \multicolumn{3}{|c|}{ H1 } & \multicolumn{3}{|c|}{ H2 } & & \multicolumn{3}{|c|}{ D1 } & \multicolumn{3}{|c|}{ D2 } & \multirow[t]{2}{*}{$\mathbf{P}$} \\
\hline & & 0 & 1 & 2 & $\mathbf{0}$ & 1 & 2 & & $\mathbf{0}$ & 1 & 2 & 0 & 1 & 2 & \\
\hline \multirow[t]{2}{*}{$\begin{array}{l}\text { Mesial } \\
\text { papilla }\end{array}$} & $\begin{array}{c}6 \\
\text { months }\end{array}$ & $\begin{array}{c}1 \\
(10 \%)\end{array}$ & $\begin{array}{c}5 \\
(50 \%)\end{array}$ & $\begin{array}{c}4 \\
(40 \%)\end{array}$ & $\begin{array}{c}1 \\
(10 \%)\end{array}$ & $\begin{array}{c}4 \\
(40 \%)\end{array}$ & $\begin{array}{c}5 \\
(50 \%)\end{array}$ & .89 & $\begin{array}{c}1 \\
(10 \%)\end{array}$ & $\begin{array}{c}6 \\
(60 \%)\end{array}$ & $\begin{array}{c}3 \\
(30 \%)\end{array}$ & $\begin{array}{c}1 \\
(10 \%)\end{array}$ & $\begin{array}{c}5 \\
(50 \%)\end{array}$ & $\begin{array}{c}4 \\
(40 \%)\end{array}$ & .89 \\
\hline & $\begin{array}{c}24 \\
\text { months }\end{array}$ & $\begin{array}{c}2 \\
(20 \%)\end{array}$ & $\begin{array}{c}6 \\
(60 \%)\end{array}$ & $\begin{array}{c}2 \\
(20 \%)\end{array}$ & $\begin{array}{c}2 \\
(20 \%)\end{array}$ & $\begin{array}{c}5 \\
(50 \%)\end{array}$ & $\begin{array}{c}3 \\
(30 \%)\end{array}$ & .86 & $\begin{array}{c}3 \\
(30 \%)\end{array}$ & $\begin{array}{c}7 \\
(70 \%)\end{array}$ & $\begin{array}{c}0 \\
(0 \%)\end{array}$ & $\begin{array}{c}2 \\
(20 \%)\end{array}$ & $\begin{array}{c}6 \\
(60 \%)\end{array}$ & $\begin{array}{c}2 \\
(20 \%)\end{array}$ & .32 \\
\hline \multirow[t]{2}{*}{ Distal papilla } & $\begin{array}{c}6 \\
\text { months }\end{array}$ & $\begin{array}{c}1 \\
(10 \%)\end{array}$ & $\begin{array}{c}5 \\
(50 \%)\end{array}$ & $\begin{array}{c}4 \\
(40 \%)\end{array}$ & $\begin{array}{c}1 \\
(10 \%)\end{array}$ & $\begin{array}{c}3 \\
(30 \%)\end{array}$ & $\begin{array}{c}6 \\
(60 \%)\end{array}$ & .63 & $\begin{array}{c}1 \\
(10 \%)\end{array}$ & $\begin{array}{c}5 \\
(50 \%)\end{array}$ & $\begin{array}{c}4 \\
(40 \%)\end{array}$ & $\begin{array}{c}1 \\
(10 \%)\end{array}$ & $\begin{array}{c}5 \\
(50 \%)\end{array}$ & $\begin{array}{c}4 \\
(40 \%)\end{array}$ & 1 \\
\hline & $\begin{array}{c}24 \\
\text { months }\end{array}$ & $\begin{array}{c}2 \\
(20 \%)\end{array}$ & $\begin{array}{c}6 \\
(60 \%)\end{array}$ & $\begin{array}{c}2 \\
(20 \%)\end{array}$ & $\begin{array}{c}2 \\
(20 \%)\end{array}$ & $\begin{array}{c}6 \\
(60 \%)\end{array}$ & $\begin{array}{c}2 \\
(20 \%)\end{array}$ & 1 & $\begin{array}{c}2 \\
(20 \%)\end{array}$ & $\begin{array}{c}6 \\
(60 \%)\end{array}$ & $\begin{array}{c}2 \\
(20 \%)\end{array}$ & $\begin{array}{c}2 \\
(20 \%)\end{array}$ & $\begin{array}{c}6 \\
(60 \%)\end{array}$ & $\begin{array}{c}2 \\
(20 \%)\end{array}$ & 1 \\
\hline \multirow{2}{*}{$\begin{array}{c}\text { Curvature } \\
\text { of facial } \\
\text { mucosa }\end{array}$} & $\begin{array}{c}6 \\
\text { months }\end{array}$ & $\begin{array}{c}0 \\
(0 \%)\end{array}$ & $\begin{array}{c}5 \\
(50 \%)\end{array}$ & $\begin{array}{c}5 \\
(50 \%)\end{array}$ & $\begin{array}{c}1 \\
(10 \%)\end{array}$ & $\begin{array}{c}2 \\
(20 \%)\end{array}$ & $\begin{array}{c}7 \\
(70 \%)\end{array}$ & .27 & $\begin{array}{c}0 \\
(0 \%)\end{array}$ & $\begin{array}{c}4 \\
(40 \%)\end{array}$ & $\begin{array}{c}6 \\
(60 \%)\end{array}$ & $\begin{array}{c}1 \\
(10 \%)\end{array}$ & $\begin{array}{c}4 \\
(40 \%)\end{array}$ & $\begin{array}{c}5 \\
(50 \%)\end{array}$ & .58 \\
\hline & $\begin{array}{c}24 \\
\text { months }\end{array}$ & $\begin{array}{c}1 \\
(10 \%)\end{array}$ & $\begin{array}{c}5 \\
(50 \%)\end{array}$ & $\begin{array}{c}4 \\
(40 \%)\end{array}$ & $\begin{array}{c}1 \\
(10 \%)\end{array}$ & $\begin{array}{c}4 \\
(40 \%)\end{array}$ & $\begin{array}{c}5 \\
(50 \%)\end{array}$ & .89 & $\begin{array}{c}1 \\
(10 \%)\end{array}$ & $\begin{array}{c}5 \\
(50 \%)\end{array}$ & $\begin{array}{c}4 \\
(40 \%)\end{array}$ & $\begin{array}{c}2 \\
(20 \%)\end{array}$ & $\begin{array}{c}4 \\
(40 \%)\end{array}$ & $\begin{array}{c}4 \\
(40 \%)\end{array}$ & .8 \\
\hline \multirow[t]{2}{*}{$\begin{array}{c}\text { Level of } \\
\text { facial mucosa }\end{array}$} & $\begin{array}{c}6 \\
\text { months }\end{array}$ & $\begin{array}{c}0 \\
(0 \%)\end{array}$ & $\begin{array}{c}5 \\
(50 \%)\end{array}$ & $\begin{array}{c}5 \\
(50 \%)\end{array}$ & $\begin{array}{c}1 \\
(10 \%)\end{array}$ & $\begin{array}{c}4 \\
(40 \%)\end{array}$ & $\begin{array}{c}5 \\
(50 \%)\end{array}$ & .89 & $\begin{array}{c}0 \\
(0 \%)\end{array}$ & $\begin{array}{c}7 \\
(70 \%)\end{array}$ & $\begin{array}{c}3 \\
(30 \%)\end{array}$ & $\begin{array}{c}1 \\
(10 \%)\end{array}$ & $\begin{array}{c}4 \\
(40 \%)\end{array}$ & $\begin{array}{c}5 \\
(50 \%)\end{array}$ & .65 \\
\hline & $\begin{array}{c}24 \\
\text { months }\end{array}$ & $\begin{array}{c}2 \\
(20 \%)\end{array}$ & $\begin{array}{c}6 \\
(60 \%)\end{array}$ & $\begin{array}{c}2 \\
(20 \%)\end{array}$ & $\begin{array}{c}2 \\
(20 \%)\end{array}$ & $\begin{array}{c}6 \\
(60 \%)\end{array}$ & $\begin{array}{c}2 \\
(20 \%)\end{array}$ & 1 & $\begin{array}{c}3 \\
(30 \%)\end{array}$ & $\begin{array}{c}6 \\
(60 \%)\end{array}$ & $\begin{array}{c}1 \\
(10 \%)\end{array}$ & $\begin{array}{c}2 \\
(20 \%)\end{array}$ & $\begin{array}{c}7 \\
(70 \%)\end{array}$ & $\begin{array}{c}1 \\
(10 \%)\end{array}$ & .71 \\
\hline
\end{tabular}




\begin{tabular}{|c|c|c|c|c|c|c|c|c|c|c|c|c|c|c|c|}
\hline \multirow{2}{*}{\begin{tabular}{|c} 
Root \\
convexity/ \\
soft tissue \\
color and \\
texture
\end{tabular}} & $\begin{array}{c}6 \\
\text { months }\end{array}$ & $\begin{array}{c}0 \\
(0 \%)\end{array}$ & $\begin{array}{c}2 \\
(20 \%)\end{array}$ & $\begin{array}{c}8 \\
(80 \%)\end{array}$ & $\begin{array}{c}0 \\
(0 \%)\end{array}$ & $\begin{array}{c}3 \\
(30 \%)\end{array}$ & $\begin{array}{c}7 \\
(70 \%)\end{array}$ & 6 & $\begin{array}{c}0 \\
(0 \%)\end{array}$ & $\begin{array}{c}4 \\
(40 \%)\end{array}$ & $\begin{array}{c}6 \\
(60 \%)\end{array}$ & $\begin{array}{c}0 \\
(0 \%)\end{array}$ & $\begin{array}{c}4 \\
(40 \%)\end{array}$ & $\begin{array}{c}6 \\
(60 \%)\end{array}$ & 1 \\
\hline & $\begin{array}{c}24 \\
\text { months }\end{array}$ & $\begin{array}{c}0 \\
(0 \%)\end{array}$ & $\begin{array}{c}2 \\
(20 \%)\end{array}$ & $\begin{array}{c}8 \\
(80 \%)\end{array}$ & $\begin{array}{c}0 \\
(0 \%)\end{array}$ & $\begin{array}{c}5 \\
(50 \%)\end{array}$ & $\begin{array}{c}5 \\
(50 \%)\end{array}$ & .16 & & $\begin{array}{c}6 \\
(60 \%)\end{array}$ & $\begin{array}{c}4 \\
(40 \%)\end{array}$ & $\begin{array}{c}0 \\
(0 \%)\end{array}$ & $\begin{array}{c}3 \\
(30 \%)\end{array}$ & $\begin{array}{c}7 \\
(70 \%)\end{array}$ & .17 \\
\hline \multirow[t]{2}{*}{ PES } & $\begin{array}{c}6 \\
\text { months }\end{array}$ & \multicolumn{3}{|c|}{$7.4 \pm 2$} & \multicolumn{3}{|c|}{$7.6 \pm 1.9$} & .23 & \multicolumn{3}{|c|}{$7 \pm 1.6$} & \multicolumn{3}{|c|}{$7 \pm 1.15$} & 1 \\
\hline & $\begin{array}{c}24 \\
\text { months }\end{array}$ & \multicolumn{3}{|c|}{$6 \pm 1.7$} & \multicolumn{3}{|c|}{$6.1 \pm 1.8$} & .89 & \multicolumn{3}{|c|}{$5.8 \pm 1.3$} & \multicolumn{3}{|c|}{$5.2 \pm 1.6$} & .19 \\
\hline \multirow[t]{2}{*}{ Tooth form } & $\begin{array}{c}6 \\
\text { months }\end{array}$ & $\begin{array}{c}0 \\
(0 \%)\end{array}$ & $\begin{array}{c}3 \\
(30 \%)\end{array}$ & $\begin{array}{c}7 \\
(70 \%)\end{array}$ & $\begin{array}{c}0 \\
(0 \%)\end{array}$ & $\begin{array}{c}5 \\
(50 \%)\end{array}$ & $\begin{array}{c}5 \\
(50 \%)\end{array}$ & .36 & $\begin{array}{c}0 \\
(0 \%)\end{array}$ & $\begin{array}{c}4 \\
(40 \%)\end{array}$ & $\begin{array}{c}6 \\
(60 \%)\end{array}$ & $\begin{array}{c}0 \\
(0 \%)\end{array}$ & $\begin{array}{c}6 \\
(60 \%)\end{array}$ & $\begin{array}{c}4 \\
(40 \%)\end{array}$ & .36 \\
\hline & $\begin{array}{c}24 \\
\text { months }\end{array}$ & $\begin{array}{c}0 \\
(0 \%)\end{array}$ & $\begin{array}{c}4 \\
(40 \%)\end{array}$ & $\begin{array}{c}6 \\
(60 \%)\end{array}$ & $\begin{array}{c}0 \\
(0 \%)\end{array}$ & $\begin{array}{c}5 \\
(50 \%)\end{array}$ & $\begin{array}{c}5 \\
(50 \%)\end{array}$ & .632 & $\begin{array}{c}0 \\
(0 \%)\end{array}$ & $\begin{array}{c}4 \\
(40 \%)\end{array}$ & $\begin{array}{c}6 \\
(60 \%)\end{array}$ & $\begin{array}{c}0 \\
(0 \%)\end{array}$ & $\begin{array}{c}6 \\
(60 \%)\end{array}$ & $\begin{array}{c}4 \\
(40 \%)\end{array}$ & 1 \\
\hline \multirow{2}{*}{$\begin{array}{c}\text { Tooth } \\
\text { volume/ } \\
\text { outline }\end{array}$} & $\begin{array}{c}6 \\
\text { months }\end{array}$ & $\begin{array}{c}0 \\
(0 \%)\end{array}$ & $\begin{array}{c}2 \\
(20 \%)\end{array}$ & $\begin{array}{c}8 \\
(80 \%)\end{array}$ & $\begin{array}{c}0 \\
(0 \%)\end{array}$ & $\begin{array}{c}4 \\
(40 \%)\end{array}$ & $\begin{array}{c}6 \\
(60 \%)\end{array}$ & .06 & $\begin{array}{c}0 \\
(0 \%)\end{array}$ & $\begin{array}{c}3 \\
(30 \%)\end{array}$ & $\begin{array}{c}7 \\
(70 \%)\end{array}$ & $\begin{array}{c}0 \\
(0 \%)\end{array}$ & $\begin{array}{c}5 \\
(50 \%)\end{array}$ & $\begin{array}{c}5 \\
(50 \%)\end{array}$ & .36 \\
\hline & $\begin{array}{c}24 \\
\text { months }\end{array}$ & $\begin{array}{c}0 \\
(0 \%)\end{array}$ & $\begin{array}{c}3 \\
(30 \%)\end{array}$ & $\begin{array}{c}7 \\
(70 \%)\end{array}$ & $\begin{array}{c}0 \\
(0 \%)\end{array}$ & $\begin{array}{c}4 \\
(40 \%)\end{array}$ & $\begin{array}{c}6 \\
(60 \%)\end{array}$ & .17 & $\begin{array}{c}0 \\
(0 \%)\end{array}$ & $\begin{array}{c}3 \\
(30 \%)\end{array}$ & $\begin{array}{c}7 \\
(70 \%)\end{array}$ & $\begin{array}{c}0 \\
(0 \%)\end{array}$ & $\begin{array}{c}5 \\
(50 \%)\end{array}$ & $\begin{array}{c}5 \\
(50 \%)\end{array}$ & .36 \\
\hline \multirow[t]{2}{*}{$\begin{array}{c}\text { Color (hue/ } \\
\text { value) }\end{array}$} & $\begin{array}{c}6 \\
\text { months }\end{array}$ & $\begin{array}{c}0 \\
(0 \%)\end{array}$ & $\begin{array}{c}1 \\
(10 \%)\end{array}$ & $\begin{array}{c}9 \\
(90 \%)\end{array}$ & $\begin{array}{c}0 \\
(0 \%)\end{array}$ & $\begin{array}{c}4 \\
(40 \%)\end{array}$ & $\begin{array}{c}6 \\
(60 \%)\end{array}$ & .128 & $\begin{array}{c}0 \\
(0 \%)\end{array}$ & $\begin{array}{c}1 \\
(10 \%)\end{array}$ & $\begin{array}{c}9 \\
(90 \%)\end{array}$ & $\begin{array}{c}0 \\
(0 \%)\end{array}$ & $\begin{array}{c}2 \\
(20 \%)\end{array}$ & $\begin{array}{c}8 \\
(80 \%)\end{array}$ & .53 \\
\hline & $\begin{array}{c}24 \\
\text { months }\end{array}$ & $\begin{array}{c}0 \\
(0 \%)\end{array}$ & $\begin{array}{c}1 \\
(10 \%)\end{array}$ & $\begin{array}{c}9 \\
(90 \%)\end{array}$ & $\begin{array}{c}0 \\
(0 \%)\end{array}$ & $\begin{array}{c}5 \\
(50 \%)\end{array}$ & $\begin{array}{c}5 \\
(50 \%)\end{array}$ & .092 & $\begin{array}{c}0 \\
(0 \%)\end{array}$ & $\begin{array}{c}1 \\
(10 \%)\end{array}$ & $\begin{array}{c}9 \\
(90 \%)\end{array}$ & $\begin{array}{c}0 \\
(0 \%)\end{array}$ & $\begin{array}{c}2 \\
(20 \%)\end{array}$ & $\begin{array}{c}8 \\
(80 \%)\end{array}$ & .53 \\
\hline \multirow[t]{2}{*}{$\begin{array}{l}\text { Surface } \\
\text { texture }\end{array}$} & $\begin{array}{c}6 \\
\text { months }\end{array}$ & $\begin{array}{c}0 \\
(0 \%)\end{array}$ & $\begin{array}{c}2 \\
(20 \%)\end{array}$ & $\begin{array}{c}8 \\
(80 \%)\end{array}$ & $\begin{array}{c}0 \\
(0 \%)\end{array}$ & $\begin{array}{c}3 \\
(30 \%)\end{array}$ & $\begin{array}{c}7 \\
(70 \%)\end{array}$ & .6 & $\begin{array}{c}0 \\
(0 \%)\end{array}$ & $\begin{array}{c}2 \\
(20 \%)\end{array}$ & $\begin{array}{c}8 \\
(80 \%)\end{array}$ & $\begin{array}{c}0 \\
(0 \%)\end{array}$ & $\begin{array}{c}3 \\
(30 \%)\end{array}$ & $\begin{array}{c}7 \\
(70 \%)\end{array}$ & .6 \\
\hline & $\begin{array}{c}24 \\
\text { months }\end{array}$ & $\begin{array}{c}0 \\
(0 \%)\end{array}$ & $\begin{array}{c}2 \\
(20 \%)\end{array}$ & $\begin{array}{c}8 \\
(80 \%)\end{array}$ & $\begin{array}{c}0 \\
(0 \%)\end{array}$ & $\begin{array}{c}3 \\
(30 \%)\end{array}$ & $\begin{array}{c}7 \\
(70 \%)\end{array}$ & .6 & $\begin{array}{c}0 \\
(0 \%)\end{array}$ & $\begin{array}{c}2 \\
(20 \%)\end{array}$ & $\begin{array}{c}8 \\
(80 \%)\end{array}$ & $\begin{array}{c}0 \\
(0 \%)\end{array}$ & $\begin{array}{c}3 \\
(30 \%)\end{array}$ & $\begin{array}{c}7 \\
(70 \%)\end{array}$ & .6 \\
\hline \multirow[t]{2}{*}{ Translucency } & $\begin{array}{c}6 \\
\text { months }\end{array}$ & $\begin{array}{c}2 \\
(20 \%)\end{array}$ & $\begin{array}{c}2 \\
(20 \%)\end{array}$ & $\begin{array}{c}6 \\
(60 \%)\end{array}$ & $\begin{array}{c}2 \\
(20 \%)\end{array}$ & $\begin{array}{c}2 \\
(20 \%)\end{array}$ & $\begin{array}{c}6 \\
(60 \%)\end{array}$ & 1 & $\begin{array}{c}1 \\
(10 \%)\end{array}$ & $\begin{array}{c}2 \\
(20 \%)\end{array}$ & $\begin{array}{c}7 \\
(70 \%)\end{array}$ & $\begin{array}{c}1 \\
(10 \%)\end{array}$ & $\begin{array}{c}2 \\
(20 \%)\end{array}$ & $\begin{array}{c}7 \\
(70 \%)\end{array}$ & 1 \\
\hline & $\begin{array}{c}24 \\
\text { months }\end{array}$ & $\begin{array}{c}2 \\
(20 \%)\end{array}$ & $\begin{array}{c}2 \\
(20 \%)\end{array}$ & $\begin{array}{c}6 \\
(60 \%)\end{array}$ & $\begin{array}{c}2 \\
(20 \%)\end{array}$ & $\begin{array}{c}3 \\
(30 \%)\end{array}$ & $\begin{array}{c}5 \\
(50 \%)\end{array}$ & .86 & $\begin{array}{c}1 \\
(10 \%)\end{array}$ & $\begin{array}{c}2 \\
(20 \%)\end{array}$ & $\begin{array}{c}7 \\
(70 \%)\end{array}$ & $\begin{array}{c}1 \\
(10 \%)\end{array}$ & $\begin{array}{c}2 \\
(20 \%)\end{array}$ & $\begin{array}{c}7 \\
(70 \%)\end{array}$ & 1 \\
\hline \multirow[t]{2}{*}{ WES } & $\begin{array}{c}6 \\
\text { months }\end{array}$ & \multicolumn{3}{|c|}{$8.7 \pm 1.8$} & \multicolumn{3}{|c|}{$8.2 \pm 1.9$} & .63 & \multicolumn{3}{|c|}{$8.6 \pm 1.9$} & \multicolumn{3}{|c|}{$8 \pm 2$} & .65 \\
\hline & months & \multicolumn{3}{|c|}{$8.4 \pm 1.8$} & \multicolumn{3}{|c|}{$7.3 \pm 2.3$} & .04 & \multicolumn{3}{|c|}{$8.6 \pm 1.8$} & \multicolumn{3}{|c|}{$8 \pm 2$} & .65 \\
\hline
\end{tabular}

\section{DISCUSSION}

The distinction between relative and absolute contradictions, for implant treatment, is not fully outlined and should be adapted to assorted situations. Particular patients, who display general pathoses, as diabetes, should be handled through a well-trained operating team under conditions that accurately regard the surgical protocol. Certain habits, disease, or medication may modify the healing capacity of the bone. Diabetes mellitus represents a substantially reduced healing potential while, controlled diabetes have bone with moderately reduced healing potential..$^{35}$ 
Poor bone volume and anatomic deviations are frequently met during implant placement in maxilla. In light of the concerns surrounding diabetes, bone grafting, guided tissue regeneration, and bone healing, the present study is a prospective clinical trial to compare between single tooth implant placement in anterior esthetic region in controlled type 2 diabetic and healthy patient using bone graft augmentation (xenograft) and guided tissue regeneration (resorbable).

The procedures of tissue augmentation are recommended to compensate the expected bone resorption and maintain the integrity of the osseous plate dimension in esthetic zones. An efficient regenerative protocol is the milestone to restore both the hard and soft tissue dimensions and preserve the good esthetic results over time. ${ }^{13,36}$ In the current study, xenograft bone substitute and resorbable membrane were used in both groups because the xenograft act as osteoconductive scaffold and beside that it would not resorbed during the normal bone remodeling. ${ }^{37}$ Moreover, several authors have reported that a xenograft particulate material placed into the buccal space minimizes the amount of osseous collapse..$^{38,39}$

The results of this present study revealed that PI and crestal bone loss were significantly higher in controlled diabetics than in the normal individual group.

MBL in controlled diabetic groups were significantly higher than in controlled diabetic normal groups especially after 6 months in the present study. Our results were in accordance with Mokeem et al, who reported that radiographic bone loss was increased in diabetic patients compared to healthy individuals. ${ }^{20}$

PESWES includes the assessment of the periimplant soft tissues and the implant-supported restoration to allow an inclusive esthetic assessment. Data of our study pointed out that there was no worthy variation among the study groups with regards to WES. The level of mid-facial mucosal height with mesial papillae were awarded the lowest scores for both groups, which may be caused by the mild degree of soft tissue regression that was encountered in the two groups at the end of follow up period. When counterweighting the total mean PES among the study groups, no worthy variation was detected. The lowest score for the two studied groups was linked with the color of the implant crown, which has also been reported by Cosyn et al. $(2012)^{40}$ and Raes et al. (2011). ${ }^{41}$ The color of the restoration is dependent on the process of shade matching with surrounding teeth which, in turn, based on quality and experience of the operator and the dental technician fabricating the restoration. Optimal shade matching, which would express as high WES score for color, may be difficult to realize since it depends on variety of factors as the required type and thickness of the ceramic material ${ }^{42}$ to get the desired esthetics and the shade taking device. ${ }^{43}$ Furthermore, in spite of the utilizing of shade tap as a conventional technique to record the shade of the prospective ceramic crown, this technique stills highly subjective and is obvious in our low WES for Color. ${ }^{44}$

The authors are aware that future controlled investigations with a larger number of patients in the diabetic group are needed to assure the real effect of the condition on the dental implant outcome in various clinical situations with more implants systems offering variety of surface treatments.

\section{CONCLUSIONS}

Single tooth dental implants can remain functionally and esthetically acceptable in type 2 diabetic patients in a manner similar to healthy patients provided carful controlling and maintaining of blood glycemic level. 


\section{REFERENCES}

1. Elangovan S, Avila-Ortiz G. Case Selection is Critical for Successful Outcomes Following Immediate Implant Placement in the Esthetic Zone. J Evid Based Dent Pract. 2017; $17: 135-138$.

2. Chen J, Zhang Z, Chen X, Zhang X. Influence of custommade implant designs on the biomechanical performance for the case of immediate post-extraction placement in the maxillary esthetic zone: a finite element analysis. Comput Methods BiomechBiomed Engin. 2017;20:636-644.

3. Brignardello-Petersen R. No evidence that bone thickness will affect soft-tissue and esthetic outcomes in patients with thin buccal bone undergoing immediate implant placement in the anterior maxillary zone. J Am Dent Assoc. 2017;148:e20.

4. Buser D, Chappuis V, Belser UC, Chen S. Implant placement post extraction in esthetic single tooth sites: when immediate, when early, when late? Periodontol 2000. 2017;73:84-102.

5. García-Moreno S, González-Serrano J, López-Pintor RM, Pardal-Peláez B, Hernández G, Martínez-González JM. Implant stability using piezoelectric bone surgery compared with conventional drilling: a systematic review and meta-analysis. Int J Oral Maxillofac Surg. 2018;47:14531464.

6. Glibert M, Östman S, De Bruyn H, Östman PO. The Influence of Initial Hard and Soft Tissue Dimensions on Initial Crestal Bone Loss of Immediately Loaded Dental Implants. Int J Periodontics Restorative Dent. 2018;38:873878.

7. Chien HH, Schroering RL, Prasad HS, Tatakis DN. Effects of a new implant abutment design on peri-implant soft tissues. J Oral Implantol. 2014 ;40:581-8.

8. Souza AB, Alshihri A, Kämmerer PW, Araújo MG, Gallucci GO. Histological and micro-CT analysis of peri-implant soft and hard tissue healing on implants with different healing abutments configurations. Clin Oral Implants Res. 2018;29:1007-1015.

9. Barroso-Panella A, Gargallo-Albiol J, Hérnandez-Alfaro F. Evaluation of Bone Stability and Esthetic Results After Immediate Implant Placement Using a Novel Synthetic Bone Substitute in the Anterior Zone: Results After 12 Months. Int J Periodontics Restorative Dent. 2018;38:235243

10. Jiang X, Zhang Y, Di P, Lin Y. Hard tissue volume stability of guided bone regeneration during the healing stage in the anterior maxilla: A clinical and radiographic study. Clin Implant Dent Relat Res. 2018;20:68-75.
11. Ko YC, Huang HL, Shen YW, Cai JY, Fuh LJ, Hsu JT. Variations in crestal cortical bone thickness at dental implant sites in different regions of the jawbone. Clin Implant Dent Relat Res. 2017;19:440-446.

12. Kuchler U, Chappuis V, Gruber R, Lang NP, Salvi GE. Immediate implant placement with simultaneous guided bone regeneration in the esthetic zone: 10-year clinical and radiographic outcomes. Clin Oral Implants Res. 2016;27:253-7.

13. Hof M, Pommer B, Ambros H, Jesch P, Vogl S, Zechner W. Does Timing of Implant Placement Affect Implant Therapy Outcome in the Aesthetic Zone? A Clinical, Radiological, Aesthetic, and Patient-Based Evaluation. Clin Implant Dent Relat Res. 2015;17:1188-99.

14. Gómez-de Diego R, Mang-de la Rosa Mdel R, RomeroPérez MJ, Cutando-Soriano A, López-Valverde-Centeno A. Indications and contraindications of dental implants in medically compromised patients: update. Med Oral Patol Oral Cir Bucal. 2014 1;19:e483-9.

15. Chrcanovic BR, Kisch J, Albrektsson T, Wennerberg A. Analysis of risk factors for cluster behavior of dental implant failures. Clin Implant Dent Relat Res. 2017;19: 632-642.

16. Annibali S, Pranno N, Cristalli MP, La Monaca G, Polimeni A. Survival Analysis of Implant in Patients With Diabetes Mellitus: A Systematic Review. Implant Dent. 2016;25:663-74.

17. Hurst D. Evidence unclear on whether Type I or II diabetes increases the risk of implant failure. Evid Based Dent. 2014;15:102-3.

18. Chrcanovic BR, Albrektsson T, Wennerberg A. Diabetes and oral implant failure: a systematic review. J Dent Res. 2014;93:859-67.

19. Oates TW, Huynh-Ba G, Vargas A, Alexander P, Feine J. A critical review of diabetes, glycemic control, and dental implant therapy. Clin Oral Implants Res. 2013;24:117-27.

20. Mokeem S, Alfadda SA, Al-Shibani N, Alrabiah M, AlHamdan RS, Vohra F, Abduljabbar T. Clinical and radiographic peri-implant variables around short dental implants in type 2 diabetic, prediabetic, and non-diabetic patients. Clin Implant Dent Relat Res. 2018 Nov 9.

21. Yadav R, Agrawal KK, Rao J, Anwar M, Alvi HA, Singh K, Himanshu D. Crestal Bone Loss under Delayed Loading of Full Thickness Versus Flapless Surgically Placed Dental Implants in Controlled Type 2 Diabetic Patients: A Parallel Group Randomized Clinical Trial. J Prosthodont. 2018;27:611-617. 
22. Ormianer Z, Block J, Matalon S, Kohen J. The Effect of Moderately Controlled Type 2 Diabetes on Dental Implant Survival and Peri-implant Bone Loss: A Long-Term Retrospective Study. Int J Oral Maxillofac Implants. 2018;33:389-394.

23. Hegazi R, El-Gamal M, Abdel-Hady N, Hamdy O. Epidemiology of and Risk Factors for Type 2 Diabetes in Egypt. Ann Glob Health. 2015;81:814-20.

24. Eskow CC, Oates TW. Dental Implant Survival and Complication Rate over 2 Years for Individuals with Poorly Controlled Type 2 Diabetes Mellitus. Clin Implant Dent Relat Res. 2017;19:423-431.

25. de Araújo Nobre M, Maló P, Gonçalves Y, Sabas A, Salvado F. Dental implants indiabetic patients: retrospective cohort study reporting on implant survival and risk indicators for excessive marginal bone loss at 5 years. J Oral Rehabil. 2016;43:863-870.

26. Moraschini V, Barboza ES, Peixoto GA. The impact of diabetes on dental implant failure: a systematic review and metaanalysis. Int J Oral Maxillofac Surg. 2016 ;45:1237-45.

27. Dŏgan ŞB, Kurtiş MB, Tüter G, Serdar M, Watanabe K, Karakış S. Evaluation of Clinical Parameters and Levels of Proinflammatory Cytokines in the Crevicular Fluid Around Dental Implants in Patients with Type 2 Diabetes Mellitus. Int J Oral Maxillofac Implants. 2015;30(5):1119-27.

28. Alasqah MN, Alrabiah M, Al-Aali KA, Mokeem SA, Binmahfooz AM, ArRejaie AS, Abduljabbar T. Peri-implant soft tissue status and crestal bone levels around adjacent implants placed in patients with and without type- 2 diabetes mellitus: 6 years follow-up results. Clin Implant Dent Relat Res. 2018;20:562-568.

29. Chrcanovic BR, Kisch J, Albrektsson T, Wennerberg A. Factors Influencing Early Dental Implant Failures. J Dent Res. 2016 ;95:995-1002.

30. Albrektsson T, Zarb G. Current interpretations of the osseointegrated response: Clinical Significance. Int j Prosthodont 1993; 6: 95-105.

31. Kniha K, Milz S, Kniha H, Ayoub N, Hölzle F, Modabber A. Peri-implant Crestal Bone Changes Around Zirconia Implants in Periodontally Healthy and Compromised Patients. Int J Oral Maxillofac Implants. 2018;33:217-222

32. Gu YX, Shi JY, Zhuang LF, Qiao SC, Xu YY, Lai HC. Esthetic outcome and alterations of soft tissue around single implant crowns: a 2-year prospective study. Clin Oral Implants Res. 2015;26:909-914.

33. Migliorati M, Amorfini L, Signori A, Biavati A S, Benedicenti S. Clinical and Aesthetic Outcome with Post-Extractive Implants with or without Soft Tissue Augmentation:
A 2-Year Randomized. Clinical Trial. Clin Implant Dent Relat Res. 2015;17:983-95.

34. Fürhauser R, Florescu D, Benesch T, Haas R, Mailath G, Watzek G. Evaluation of soft tissue around single-tooth implant crowns: the pink esthetic score. Clin Oral Implants Res 2005; 16:639-644.

35. Tagliareni J, Clarkson E. Basic Concepts and Techniques of Dental Implants. Dent Clin N Am. 2015;59 : 255-264.

36. Santing HJ, Raghoebar GM, Vissink A, den Hartog L, Meijer HJ. Performance of the Straumann Bone Level Implant system for anterior single-tooth replacements in augmented and nonaugmented sites: a prospective cohort study with 60 consecutive patients. Clin Oral Implants Res. 2013;24:941-8.

37. Jensen SS, Bosshardt DD, Gruber R, Buser D. Long-term stability of contour augmentation in the esthetic zone: histologic and histomorphometric evaluation of 12 human biopsies 14 to 80 months after augmentation. J Periodontol. 2014;85:1549-56.

38. ArRejaie A, Al-Harbi F, Alagl AS, Hassan KS. PlateletRich Plasma Gel Combined with Bovine-Derived Xenograft for the Treatment of Dehiscence Around Immediately Placed Conventionally Loaded Dental Implants in Humans: Cone Beam Computed Tomography and ThreeDimensional Image Evaluation. Int J Oral Maxillofac Implants. 2016;31:431-8.

39. Mannai C. Early implant loading in severely resorbed maxilla using xenograft, autograft, and platelet-rich plasma in 97 patients. J Oral Maxillofac Surg. 2006 ;64:1420-6.

40. Cosyn J, Eghbali A, De Bruyn H, Dierens M, De Rouck T. Single Implant Treatment in Healing Versus Healed Sites of the Anterior Maxilla: An Aesthetic Evaluation. Clin Implant Dent Relat Res 2012;14:517-26.

41. Raes F, Cosyn J, Crommelinck E, Coessens P, De Bruyn H. Immediate and conventional single implant treatment in the anterior maxilla: 1-year results of a case series on hard and soft tissue response and aesthetics. J Clin Periodontol 2011;38:385-394.

42. Wee AG, Monaghan P, Johnston WM. Variation in color between intended matched shade and fabricated shade of dental porcelain. J Prosthet Dent. 2002 ;87:657-66.

43. Li Q, Wang YN. Comparison of shade matching by visual observation and an intraoral dental colorimeter. J Oral Rehabil 2007;34:848-854.

44. Miller L. Organizing color in dentistry. J Am Dent Assoc 1987;9: 26-40. 